XXXII. Kongress der Schweizerischen Gesellschaft für Dermatologie und Venereologie vom 14. und 15. Oktober 1950 in Zürich

Dermatologica. 1951;102:317-318

\title{
I. Demonstrationen - Démonstrations
}

\begin{tabular}{|l|l|l} 
W. & Lutz \\
\hline
\end{tabular}

Basel

1. Mycosis fungoides im Stadium multipler Granulome:

Frau Wer., 1875, vor einem Jahr noch hartnäckige flache, schuppende, unscharf

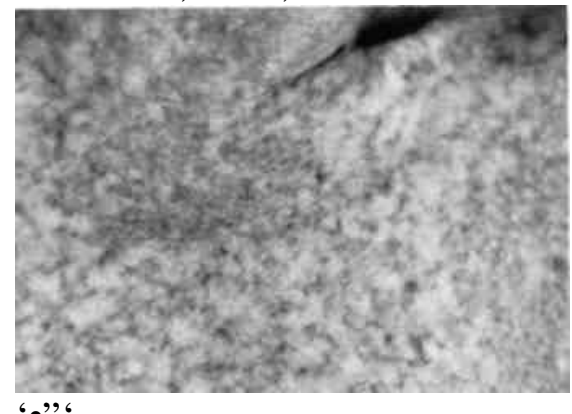

Abb. 1
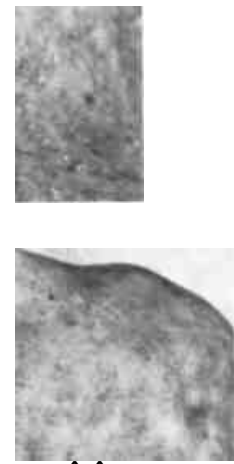

$\mathrm{j} Æ \hat{E} \hat{E}$

Abb. 2

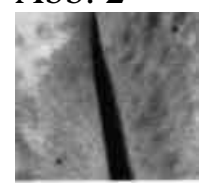

$\mathrm{J}$

Dermatologica. Vol. 102. No. 4/6 (1951

21

318

Demonstrationen - Demonstrations 
begrenzte, größere und kleinere, zum Teil ekzematoide Plaques, in kurzer Zeit Entwicklung der Granulome.

2. Mycosis fungoídes:

Frau Mö., 1893, 1946 stärkere seborrhoische Schuppung mit leichter Haut-reizung auf dem behaarten Kopf; 1948 etwas uncharakteristische, leicht psoriasi-forme Dermatose über den ganzen Körper; jetzt bietet die Haut ein Bild, das mit seiner retikulären Zeichnung etwas Poikilodermieartiges aufweist (Abb. 1).

3. Pustulosis varioliformis Kaposi:

28jährige Frau mit einem seit Kindheit bestehenden chronisch-konstitutionellen Ekzem.

4. Sehr ausgedehntes, fixes Arzneiexanthem nach Optalidon: Sehr intensive und sehr persistente Pigmentierung nach Abklingen des Erythems (Abb. 2).

Prof. P. ROBERT, Bern:

1. Akutes Gesíchtsekzem infolge Überempfindlichkeit auf Chrysanthemum carna-tum (Sonnenlicht)*.

Pat. S. G., geb. 1906.

Famílíenanamnese o. B. Persönliche Anamnese: Mit 10 Jahren Urticaria, später nie ernstlich krank. Jetziges Leiden: Erstmaliges Auftreten der Hautaffektion vor etwa 14 Jahren unter dem Bild eines akuten Gesichtsekzemes, welches nach Be-handlung mit Umschlägen rasch zurückzugehen pflegte. Seither sei die gleiche Hautaffektion regelmäßig jeden Herbst, seit einigen Jahren jeden Friihling und Herbst, aufgetreten. Da die Ehefrau des Patienten als Nebenverdienst Blumen verkauft, ist der Patient auf einen bestimmten Zusammenhang zwischen seinem Leiden und einer bestimmten Blumensorte aufmerksam gemacht worden. Das Gesichtsekzem trat nämlich jedesmal auf, wenn im Friihling die «gelben Margueriten» (Abb. 1) und im Herbst die Astern verkauft wurden. In den letzten Jahren wurden deshalb diese Blumen nicht mehr bestellt, und der Patient blieb frei. Der letzte Schub be-gann am 18. Juni 1950, nachdem Patient am Vortage ein Geschäft besucht hatte, in welchem ein Strauß «gelber Margueriten» vorhanden war. Letztere wurden jedoch nicht berührt. Eine berufliche Ätiologie des Gesichtsekzems konnte aus-geschlossen werden.

Hautstatus (26. Juni 1950): Gesicht diffus gerötet, geschwollen, zahlreiche Papelchen und Bläschen aufweisend. Stellenweise Nässen und krustöse Auflagerun-gen, besonders am Kinn und an der Oberlippe. An beiden Vorderarmen einzelne frische, münzengroße Ekzemherde. * Die Blume wurde durch Herrn Prof. Rytz, Botanisches Institut der Universität, in freundlicher Weise bestimmt. 